\title{
La dialisi peritoneale: una promessa non mantenuta?
}

\author{
Roberto Cocchi
}

\author{
Servizio Aziendale di Nefrologia e Dialisi \\ Ospedale Civile Santa Maria delle Croci, Ravenna
}

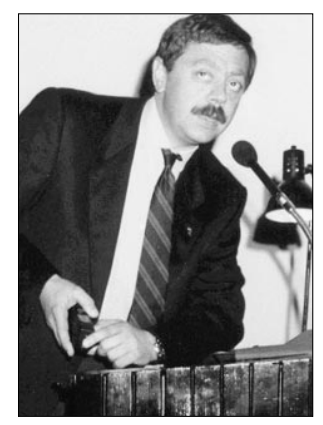

Vo orrei innanzitutto ringraziare il dr. Lombardi per avermi dato l'opportunità di esprimere il mio modesto parere sulle motivazioni del mancato decollo in Italia della dialisi peritoneale, una metodica dialitica che, superato l'impasse iniziale dell'elevata incidenza di peritoniti, prometteva di essere una valida alternativa all'emodialisi, in grado di competere con essa in termini di qualità depurativa, costi di gestione, qualità di vita ed esiti (1-4).

In effetti, l'analisi dei dati a livello mondiale evidenzia un incremento progressivo abbastanza costante dei pazienti trattati con dialisi peritoneale, mentre in Italia, nel corso degli ultimi 10-15 anni questo trend si è praticamente fermato, tanto che oggi meno di un quinto dei pazienti uremici è avviato al trattamento dialitico peritoneale e solo un decimo della popolazione dialitica è stabilmente in dialisi peritoneale.

Credo che alla base di questo parziale insuccesso, vi siano diverse ragioni che tenterò di analizzare esponendo $\mathrm{i}$ dubbi e le situazioni in cui si viene a trovare il nefrologo quando deve inserire e/o mantenere in un programma di dialisi peritoneale un paziente uremico.

Inizierò le mie considerazioni analizzando innanzitutto il "problema età" dei pazienti da avviare al trattamento sostitutivo.

Per comodità di esposizione, suddividerò la popolazione per fasce d'età in: adolescenti-giovani adulti, adultianziani "giovani" e anziani/disabili, escludendo i bambini poiché per essi la dialisi peritoneale ha confermato le aspettative dei nefrologi e le attese dei pazienti e dei loro genitori in termini di efficienza depurativa, recupero alla vita di relazione e sviluppo psicofisico, tanto che oggi essa è considerata il trattamento di elezione. Nei giovani adulti la dialisi dovrebbe essere il preludio al trapianto renale e in questo senso la peritoneale potrebbe essere il trattamento sostitutivo artificiale di prima scelta non solo per $i$ minori costi rispetto all'emodialisi, ma anche perché sono ormai numerose le segnalazioni in letteratura di una sopravvivenza dell' organo trapiantato sovrapponibile, se non migliore, nei pazienti che provengono dalla dialisi peritoneale rispetto agli emodialitici $(5,6)$. E io sono convinto che le non rare perplessità che tali pazienti esprimono al nefrologo quando si tratta di optare per la diali- si peritoneale (immagine di sé legata alla presenza del catetere peritoneale, problemi organizzativi per lo studio, lo svago e il lavoro) cadrebbero se vi fosse una buona probabilità di trapianto nell' arco di 1-2 anni. Purtroppo sappiamo tutti che l'attesa è spesso lunga e non raramente supera la sopravvivenza media della metodica che a 5 anni non eccede il 62\% (7). La APD in tal senso ha risolto alcuni di tali problemi, ma non tutti: sicuramente la metodica automatizzata riduce l'impatto della gestione dialitica e consente una migliore vita di relazione, ma non ci sono dati basati sull' evidenza che documentino una migliore sopravvivenza della metodica rispetto alla CAPD. Rimane quindi il problema di pazienti che iniziano con la dialisi peritoneale e passano poi all'emodialisi prima del trapianto e il nefrologo italiano non è culturalmente portato a scegliere un percorso discretamente complesso e a convincere $\mathrm{i}$ pazienti ad accettarlo esponendo loro in primo luogo i vantaggi che esso comporta; per cui, nel dubbio, molto spesso sceglie o fa scegliere al paziente l'emodialisi come primo trattamento.

Con l'avanzare dell' età, parlando pertanto di adulti e "giovani" anziani, si assiste a un progressivo incremento dei fattori comorbidi che riducono sensibilmente le possibilità di tra- 
pianto e accentuano ancora di più in molti nefrologi le perplessità a inserire pazienti in una metodica dialitica con una probabilità di sopravvivenza sensibilmente inferiore rispetto alla loro aspettativa di vita.

Vorrei infine affrontare il problema della scelta della metodica dialitica nel paziente anziano e/o disabile. Con la caduta pressoché completa delle controindicazioni alla dialisi, ci si scontra sempre più spesso con situazioni al limite di persone non autosufficienti da avviare al trattamento sostitutivo. La difficoltà a reperire un partner nell'ambito familiare preclude o quantomeno riduce notevolmente le possibilità di avviare tali pazienti alla dialisi peritoneale. Le uniche alternative possibili sono la dialisi peritoneale ospedaliera, che peraltro unisce a una scarsa efficienza depurativa costi di gestione particolarmente elevati, e l'internamento dei pazienti in strutture protette dove personale dedicato possa loro eseguire la dialisi peritoneale o manuale, o automatizzata.

Si tratta quasi sempre di strade "in salita" dal punto di vista organizzativo e anche umano, per cui il nefrologo opta spesso per l'emodialisi ospedaliera, tanto più che il trattamento extracorporeo è oggi talmente affidabile che consente di trattare con buona tranquillità soggetti ad alto rischio.

Un altro motivo che a mio avviso ha contribuito al parziale insuccesso della dialisi peritoneale in Italia è legato al fatto che essa non è praticamente in uso nei Centri privati, Centri che coprono una notevole fetta di trattamenti specie nel Sud del Paese. Non sono in grado di dare una motivazione valida a tale scelta del privato, così fortemente sbilanciata verso l'emodialisi, ma credo comunque che sia principalmente collegata a problemi di tipo economico.

In conclusione, le principali cause dell'insoddisfacente sviluppo della dialisi peritoneale in Italia, ritengo che possano essere così riassunte:

- numero esiguo di trapianti eseguiti annualmente in Italia;

- scarsa propensione da parte dei nefrologi e dei pazienti ad accettare un trattamento dialitico, quale quello peritoneale, di durata relativamente limitata e comunque spesso inferiore alla durata della vita biologica dei pazienti stessi;

- difficoltà di allocazione dei pazienti anziani/disabili in strutture o in sistemi organizzativi che consentano di eseguire la dialisi peritoneale;

- mancata penetrazione della dialisi peritoneale nelle strutture private.

Non è semplice trovare soluzioni valide e applicabili per tentare di superare ostacoli di tale rilevanza,

Non ho ovviamente soluzioni al problema del numero insufficiente di trapianti in Italia, né sono in grado di prevedere se e quando la ricerca ci permetterà di avere a disposizione materiali plastici e soluzioni dializzanti così biocompatibili da garantire una sopravvivenza della metodica sovrapponibile all'emodialisi.

Ritengo pertanto che i nostri sforzi dovrebbero essere mirati al raggiungimento di alcuni obiettivi realmente raggiungibili. In particolare è indispensabile che entri nella mentalità nefrologica che la dialisi peritoneale è un ottimo sistema per iniziare il trattamento dialitico per una serie di valide ragioni: consente una depurazione ottimale, almeno finché la diuresi è presente, preserva la funzionalità renale residua, assicura una buona qualità di vita, ha costi sensibilmente più bassi dell'emodialisi, è un ottimo trattamento dialitico preparatorio al trapianto e, infine, la sua esecuzione non compromette per nulla l'eventuale successivo trasferimento all'emodialisi se e quando i risultati non fossero compatibili agli obiettivi e agli standard prefissati. Un indispensabile corollario è che è inutile, anzi controproducente, insistere con la dialisi peritoneale là dove i risultati, in termini di depurazione e di riabilitazione, non siano più soddisfacenti. Questo atteggiamento mentale di "agilità" nel muoversi fra le varie opzioni offerte dalla terapia sostitutiva è alla base dei programmi di dialisi integrata (Fig. 1), programmi tanto più realizzabili, quanto più vengono potenziate le strutture organizzative del follow-up nefrologico predialitico (8). Se correttamente applicato, un sistema integrato di gestione del trat-

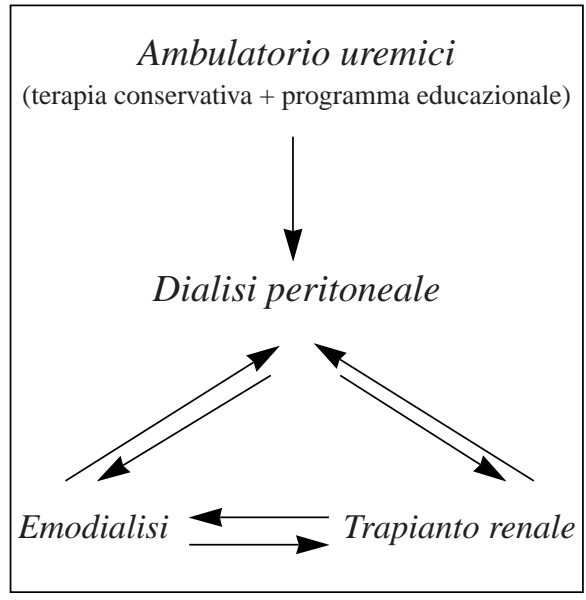

Fig. 1 - Schema di un programma integrato di ge stione della terapia dell'IRC.

tamento sostitutivo renale si è dimostrato in grado di ottenere risultati migliori, in termini di sopravvivenza, rispetto a quelli raggiungibili quando le due metodiche dialitiche sono mantenute separate e senza interscambio fra di esse (9).

Mi preme ora affrontare un altro problema che in qualche modo si collega con la necessità di non considerare come un evento drammatico il trasferimento del paziente ad altra metodica dialitica. Mi riferisco in questo caso al trasferimento temporaneo a emodialisi per perdita del catetere peritoneale. Parlando con i colleghi ho avuto a volte la sensazione che la rimozione del catetere peritoneale, a esempio per flogosi del tunnel o per peritonite non responsiva, porti con eccessiva facilità alla chiusura definitiva della dialisi peritoneale. Spesso in questi casi è sufficiente allontanarsi dall'evento di poche settimane, procedendo poi al riposizionamento del catetere e al successivo ripristino della dialisi peritoneale stessa.

Per quanto riguarda il problema della gestione della dialisi peritoneale negli anziani non autosufficienti, è indispensabile si giunga al più presto a soluzioni percorribili dal punto di vista pratico con una più intensa collaborazione con i Servizi sul territorio per fornire a casa dei pazienti, o presso struttu- 
re protette, mezzi e personale per una gestione dialitica extraospedaliera, poiché è sempre più assillante la domanda di dialisi in tali pazienti problematici.

Rimane aperto infine il problema del potenziamento della dialisi peritoneale nelle strutture private, argomento sul quale non ho risposte ma che spero venga dibattuto in questa sede poiché credo che la struttura sanitaria abbia potenzialità inespresse che, se correttamente utilizzate, potrebbero contribuire fattivamente al corretto sviluppo della dialisi peritoneale nel nostro Paese.

ranefro@auslra.village.telecomitalia.it

\section{BIBLIOGRAFIA}

1.

Burton PR, Walls J. Selectionadjusted comparison of life expectancy of patients on continuous peritoneal dialysis, haemodialysis and renal transplant. Lancet 1987; 2: 1115-9.

2. Maiorca R, Vonesh EF, Cavalli $\mathrm{P}$, et al. A multi-center selection adjusted comparison of patients and technique survivals on CAPD and hemodialysis. Perit Dial Bull 1991; 11: 118-27.

3. Piccoli G, Salomone M, Quarello $\mathrm{F}$ et al. Regional registry of dialysis and transplantation of Piedmont, Italy /RPDT. Thirteen years of experience. Nephrol Dial Transplant 1995; 10: 444-7.

4. Tucker CM, Ziller RC, Smith WR, et al. Quality of life of patients on in-center hemodialysis versus continuous ambulatory peritoneal dialysis. Perit Dial Int 1991; 11: 341-6.

5. Maiorca R, Sandrini S, Cancarini GC, et al. Integration of peritoneal dialysis and transplantation programmes. Perit Dial Int 1997; 17 (suppl.): S170-4.

6. Bleyer AJ, Burkart JM, Russel GB, Adams PL. PD patients have better outcomes in the first week after cadaveric transplantation than HD patients. Perit Dial Int 1998; 18 (suppl.): S47.

7. Lupo A, Tarchini R, Cancarini $\mathrm{GC}$, et al. Long-term outcome in continuous peritoneal dialysis: A 10 year survey by the Italian Cooperative Peritoneal Study Group. Am J Kidney Dis 1994; 24: 826-37.

8. Coles GA, Williams JD. What is the place of peritoneal dialysis in the integrated treatment of renal failure? Kidney Int 1998; 54: 2234-40.

9. Van Biesen W, DeVogeleere P, Vijt D, Vanholder R, Lameire N. Integrated care can improve long-term survival of ESRD patients. Perit Dial Int 1998; 18: 138. 